\title{
Adaptive wireless transmission strategy for maximizing energy efficiency
}

\author{
Caixia Cai ${ }^{1,2}$, Runhe Qiu ${ }^{1,2^{*}}$ (D), Xue-Qin Jiang ${ }^{1,2}$ and Wanping $X u^{1,2}$
}

\begin{abstract}
In a traditional wireless transmission system, studies about energy efficiency (EE) are usually for a fixed transmission mode. In this paper, we propose an adaptive wireless transmission (AWT) strategy with consideration of circuit power. In this AWT strategy, the transmission mode is switched between the direct transmission (DT) mode and the two-way relay transmission (TWRT) mode. The switch strategy is based on a transmission rate threshold $R_{\text {th }}$ which makes the EEs of the DT mode and the TWRT mode equal. Furthermore, we propose a transmission rate threshold determining (TRTD) algorithm with a bisection method to find the threshold $R_{\text {th }}$. The simulation results also show that our AWT strategy has the maximum $\mathrm{EE}$ at a reasonable range of transmission rate.
\end{abstract}

Keywords: Energy efficiency (EE), Direct transmission (DT), Two-way relay transmission (TWRT), Circuit power, Adaptive wireless transmission (AWT) strategy

\section{Introduction}

Energy consumption in wireless transmission system has been continuously increasing to cater for the explosive growth in demand for high-data-rate wireless applications and a wide variety of diverse quality of service (QoS) requirements during the last decade. Nowadays, the wireless terminals are usually powered by batteries. It is known that high-level energy consumption has a profound influence on the wireless terminals due to the limit of battery capacity. Therefore, it is very important to reduce energy consumption of the wireless terminals and increase energy efficiency (EE) [1-3]. Recently, a lot of advanced wireless communication techniques, such as relay technique $[4,5]$ and small cells $[6]$, have been adopted to provide a significant capacity improvement and reduce the energy consumption. In order to further reduce the energy consumption, there is also a lot of research work focusing on the optimal power allocation $[7,8]$.

A relay communication system, in which the relay forwards the signal transmitted from a source node to a

\footnotetext{
*Correspondence: qiurh@dhu.edu.cn

${ }^{1}$ College of Information Sciences and Technology, Donghua University, Renmin North, 201620 Shanghai, People's Republic of China

${ }^{2}$ Engineering Research Center of Digitized Textile \& Fashion Technology, Ministry of Education, Donghua University, Renmin North, 201620 Shanghai, People's Republic of China
}

destination node, has attracted a lot of attention, due to its ability in expanding the coverage, increasing the capacity, and reducing the power consumption. Two-way relay communication is a promising spectral-efficient transmission protocol for it only needs two time slots to complete a process of signal exchange $[9,10]$. In such a communication technique, two source nodes exchange signals with the help of relay(s). As a result, there are two traffic flows in a two-way relay transmission (TWRT) process and they are supported by the same physical channels concurrently, which enhances spectral efficiency (SE) [11].

Most current studies on the two-way relay technique mainly focus on the relay schemes, relay selection, and resource allocation from the perspective of SE [12]. However, there are less research work focusing on EE. In [13], authors studied maximizing aggregated EE utility while provisioning proportional fairness. The power allocation schemes to improve the EE in multiuser multi-carrier two-way relay networks have been designed in [14]. The issue of resource allocation problem in orthogonal frequency division multiple access (OFDMA) two-way relay networks has been considered in [15]. Its objective is to minimize the total transmit power to improve EE. However, in these research works, only transmit power has been considered. Actually, in practical wireless transmission system, energy consumption does not only include 
transmit power but also include circuit power for nonideal transmitter [16]. In [17], it has been demonstrated that the circuit power is consumed by signal processing and the device working in the active mode. The authors in [18] designed an optimal power allocation scheme to maximize EE for two-way amplify and forward (AF) relay networks with consideration of circuit power. Furthermore, the discussions above mainly focus on a fixed transmission mode, in which EE is not always the maximum at a range of transmission rate.

In this paper, to improve EE, we propose an adaptive wireless transmission (AWT) strategy in which the transmission mode is switched between the direct transmission (DT) mode and the TWRT mode. The switch strategy is based on a transmission rate threshold $R_{\text {th }}$, which makes EEs of the DT mode and the TWRT mode equal. However, the traditional metric for EE is not suitable for the subsequent analysis, and the certain threshold $R_{\text {th }}$ cannot be found. In this work, we first investigate the transmission rates of the DT mode and the TWRT mode when the scenario of two source nodes exchanging signals is considered. Then, energy consumption ratio (ECR) which is used to evaluate EE of various kinds of transmission modes is introduced with consideration of the circuit power. The ECR is defined as minimum power consumption of unit transmission rate. Finally, we propose a transmission rate threshold determining (TRTD) algorithm with a bisection method to find the threshold $R_{\mathrm{th}}$.

The main contribution of this paper can be summarized as follows:

- We give a detailed analysis of ECRs with consideration of the circuit power.

- We propose the AWT strategy in which the transmission mode is switched between the DT mode and the TWRT mode based on the threshold $R_{\mathrm{th}}$. With this strategy, it can be seen that EE is always the maximum at a range of transmission rate.
- We also propose the TRTD algorithm with a bisection method to find the threshold $R_{\mathrm{th}}$.

The remainder of this paper is as follows. Section 2 describes the system model. Section 3 introduces the power consumption and the metric of EE. Comparison and analysis of EE are presented in Section 4. Simulation results are presented in Section 5, followed by the conclusions in Section 6.

Notation: $y_{(\cdot)}$ denotes received signal at relay and source nodes. The transmission rate of source nodes $S_{1}$ and $S_{2}$ are denoted by $R_{s_{1}}$ and $R_{s_{2}}$. $R_{\mathrm{s}}$ denotes the sum transmission rate. The ECRs of the DT mode and the TWRT mode are denoted by $\eta_{\mathrm{d}}$ and $\eta_{\mathrm{t}}$. The ECR of the AWT strategy is defined by $\eta_{\mathrm{a}}=\min \left\{\eta_{\mathrm{d}}, \eta_{\mathrm{t}}\right\}$. The EEs of the DT mode and the TWRT mode are denoted by $e_{\mathrm{d}}$ and $e_{\mathrm{t}}$. The EE of the AWT strategy is defined by $e_{\mathrm{a}}=\max \left\{e_{\mathrm{d}}, e_{\mathrm{t}}\right\}$. $w_{i} \sim\left(0, \sigma^{2}\right)$ denotes a zero-mean complex-valued additive white Gaussian noise (AWGN) with variance $\sigma^{2}$. The transmit power of the source nodes and the relay node are denoted by $P_{\mathrm{s}_{j}}(j=1,2)$ and $P_{\mathrm{r}}$. $P_{\mathrm{t}}$ denotes the power consumption. $P_{\mathrm{c}}$ and $P_{\text {sic }}$ denote the circuit power and self-interference cancelation power. $E_{(\cdot)}$ represents the expectation. $f(\cdot)$ denotes the primitive function, and $f^{\prime}(\cdot)$ denotes the derived function.

\section{System model}

As shown in Fig. 1, the system model consists of two source nodes $S_{1}$ and $S_{2}$ in the DT mode. There is also a fixed relay node $\mathrm{R}$ working in the AF mechanism in the TWRT mode. All the nodes are equipped with a single antenna and operate in the half-duplex mechanism. The total transmission process of signal exchange is two time slots, then there is no direct connection between the nodes $S_{1}$ and $S_{2}$ in the TWRT mode. The perfect channel state information (CSI) is available at the nodes $S_{1}$ and $S_{2}$, which can adapt their transmission rates accordingly. In this paper, we assume that the channels are symmetrical.

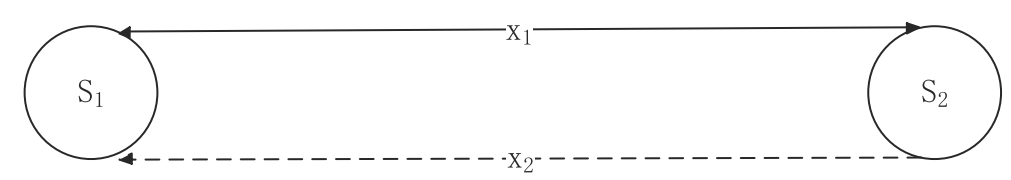

(a)

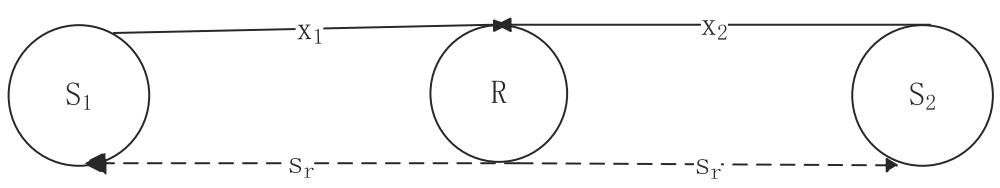

(b)

Fig. 1 The system model. a Direct transmission mode. b Two-way relay transmission mode 
The channel coefficient from the node $S_{1}$ to node $S_{2}$, the nodes $S_{1}$ and $S_{2}$ to the node $R$ is denoted by $h_{a b}, a, b \in$ $\left\{s_{1}, s_{2}, r\right\}$. The channels between any two nodes are independent. $w_{i} \sim\left(0, \sigma^{2}\right), i \in\left\{s_{j}, r\right\}(j=1,2)$, denotes a zero-mean complex-valued AWGN with variance $\sigma^{2}$. The nodes $S_{1}$ and $S_{2}$ intend to exchange their signals $x_{1}$ and $x_{2}$, respectively, with the transmit power $P_{s_{1}}$ and $P_{s_{2}}$. It is assumed that $E\left\{\left|x_{1}\right|^{2}\right\}=E\left\{\left|x_{2}\right|^{2}\right\}=1$.

\subsection{DT mode}

In the DT mode, the two source nodes $S_{1}$ and $S_{2}$ transmit signals to each other without the assistance of the relay node $R$. Two time slots are required to complete the process of signal exchange. The DT mode is shown in Fig. 1a.

In the first time slot, the source node $S_{1}$ transmits signal $x_{1}$ to the node $S_{2}$. In the second time slot, the source node $S_{2}$ transmits signal $x_{2}$ to the node $S_{1}$. The received signals $y_{s_{1}}$ and $y_{s_{2}}$ at the nodes $S_{1}$ and $S_{2}$ are, respectively, expressed as

$$
y_{s_{1}}=\sqrt{P_{s_{2}}} h_{s_{2} s_{1}} x_{2}+w_{s_{1}}
$$

and

$$
y_{s_{2}}=\sqrt{P_{s_{1}}} h_{s_{1} s_{2}} x_{1}+w_{s_{2}}
$$

where $w_{s_{1}}$ and $w_{s_{2}}$ denote the noise at the source nodes $S_{1}$ and $S_{2}$.

It is stated in [13] that the transmission rates $R_{s_{1}}$ and $R_{s_{2}}$ with unit bandwidth are

$$
R_{s_{1}}=\frac{1}{2} \log _{2}\left(1+\frac{P_{s_{2}}}{\sigma^{2}}\left|h_{s_{2} s_{1}}\right|^{2}\right)
$$

and

$$
R_{s_{2}}=\frac{1}{2} \log _{2}\left(1+\frac{P_{s_{1}}}{\sigma^{2}}\left|h_{s_{1} s_{2}}\right|^{2}\right),
$$

where coefficient $\frac{1}{2}$ accounts for the two equal time slots. We assume that $g_{s_{1} s_{2}}=\frac{\left|h_{s_{1} s_{2}}\right|^{2}}{\sigma^{2}}$, which reflects the instantaneous channel gain to noise ratio (CNR) [18] from the node $S_{1}$ to node $S_{2}$. Then, the transmission rates at the two source nodes can be expressed as

$$
R_{s_{1}}=\frac{1}{2} \log _{2}\left(1+P_{s_{2}} g_{s_{1} s_{2}}\right),
$$

and

$$
R_{s_{2}}=\frac{1}{2} \log _{2}\left(1+P_{s_{1}} g_{s_{1} s_{2}}\right) .
$$

\subsection{TWRT mode}

In a relay communication system, it can be seen that each transmission time interval (TTI) also composes of two time slot periods. In the TWRT mode, the two source nodes $S_{1}$ and $S_{2}$ transmit signal to each other through the relay node $R$. The TWRT mode is demonstrated in Fig. 1b.
In the first time slot, the two source nodes $S_{1}$ and $S_{2}$ respectively transmit signals $x_{1}$ and $x_{2}$ to the relay node $R$. The received signal $y_{r}$ at the relay node $R$ is expressed as

$$
y_{r}=\sqrt{P_{s_{1}}} h_{s_{1}} x_{1}+\sqrt{P_{s_{2}}} h_{s_{2} r} x_{2}+w_{r}
$$

where $w_{r}$ denotes the noise at the relay node $R$. We let the power of $y_{r}$ to be normalized, where $s_{r}=G y_{r}$ and

$$
G=\frac{1}{\sqrt{P_{S_{1}}\left|h_{s_{1} r}\right|^{2}+P_{S_{2}}\left|h_{s_{2} r}\right|^{2}+\sigma^{2}}} .
$$

$G$ is an amplification factor.

In the second time slot, the relay node $R$ will broadcast $s_{r}$ to the nodes $S_{1}$ and $S_{2}$ with power $P_{\mathrm{r}}$, which is the transmit power of the relay node $R$. The received signals $y_{s_{1}}{ }^{\prime}$ and $y_{S_{2}}{ }^{\prime}$ at the nodes $S_{1}$ and $S_{2}$ can be respectively expressed as

$$
y_{s_{1}}{ }^{\prime}=\sqrt{P_{\mathrm{r}}} h_{r s_{1}} s_{r}+w_{s_{1}},
$$

and

$$
y_{s_{2}}{ }^{\prime}=\sqrt{P_{\mathrm{r}}} h_{r s_{2}} s_{r}+w_{s_{2}} .
$$

Since each of the nodes receives a copy of its own transmitted signal as interference, the signal transmitted from the other source node can be decoded after selfinterference cancelation (SIC). The received signals $y_{s_{1}}$ and $y_{s_{2}}$ at the nodes $S_{1}$ and $S_{2}$ are, respectively, expressed as

$$
y_{s_{1}}=G\left(\sqrt{P_{\mathrm{r}} P_{s_{2}}} h_{r s_{1}} h_{s_{2} r} x_{2}+\sqrt{P_{\mathrm{r}}} h_{r s_{1}} w_{r}\right)+w_{s_{1}},
$$

and

$$
y_{s_{2}}=G\left(\sqrt{P_{\mathrm{r}} P_{s_{1}}} h_{r s_{2}} h_{s_{1} r} x_{1}+\sqrt{P_{\mathrm{r}}} h_{r s_{2}} w_{r}\right)+w_{s_{2}} .
$$

The transmission rates $R_{s_{1}}$ and $R_{s_{2}}$ with unit bandwidth are

$$
R_{s_{1}}=\frac{1}{2} \log _{2}\left(1+\frac{P_{\mathrm{r}} P_{s_{2}}\left|h_{r_{1}}\right|^{2}\left|h_{s_{2}}\right|^{2}}{\left(\left|h_{r_{1}}\right|^{2} P_{S_{1}}+\left|h_{s_{2}}\right|^{2}\left(P_{s_{2}}+P_{\mathrm{r}}\right)+\sigma^{2}\right) \sigma^{2}}\right),
$$

and

$$
R_{s_{2}}=\frac{1}{2} \log _{2}\left(1+\frac{P_{\mathrm{r}} P_{s_{1}}\left|h_{r_{2}}\right|^{2}\left|h_{s_{1} r}\right|^{2}}{\left(\left|h_{r s_{1}}\right|^{2}\left(P_{s_{1}}+P_{\mathrm{r}}\right)+\left|h_{s_{2}}\right|^{2} P_{s_{2}}+\sigma^{2}\right) \sigma^{2}}\right) .
$$

We assume that $g_{s_{1} r}=\frac{\left|h_{s_{1} r}\right|^{2}}{\sigma^{2}}$ and $g_{s_{2} r}=\frac{\left|h_{s_{2} r}\right|^{2}}{\sigma^{2}}$ are the CNRs from the nodes $S_{1}$ and $S_{2}$ to the node $R$, respectively. Since the channel is completely symmetrical, and $h_{r s_{1}}=h_{s_{1} r}, h_{r s_{2}}=h_{s_{2} r}$. Then, the transmission rates at the two source nodes can be respectively expressed as

$$
R_{s_{1}}=\frac{1}{2} \log _{2}\left(1+\frac{P_{\mathrm{r}} P_{s_{2}} g_{s_{1}} g_{s_{2} r}}{P_{s_{1}} g_{s_{1} r}+\left(P_{s_{2}}+P_{\mathrm{r}}\right) g_{s_{2} r}+1}\right),
$$

and 


$$
R_{s_{2}}=\frac{1}{2} \log _{2}\left(1+\frac{P_{\mathrm{r}} P_{s_{1}} g_{s_{1} r} g_{s_{2} r}}{\left(P_{s_{1}}+P_{\mathrm{r}}\right) g_{s_{1} r}+P_{s_{2}} g_{s_{2} r}+1}\right)
$$

\section{Power consumption and metric of energy efficiency}

In this section, we formulate the power consumptions and the metric of EEs in the DT mode and the TWRT mode. It is shown in [19] that during the entail transmission process, in addition to the transmit power $P_{s_{1}}$ and $P_{s_{2}}$, mobile devices also incur additional circuit power $P_{\mathrm{c}}$ which is relatively independent of the transmission rate. Therefore, the power consumptions in the DT mode and the TWRT mode are mainly composed of the transmit power and the circuit power.

\subsection{Power consumption in DT mode}

It is stated in [20] that $P_{\mathrm{c}}$ is incurred by signal processing and the device working in active mode, and it can be modeled as a linear function of the transmission rate. Then, $P_{\mathrm{c}}$ is given by $P_{\mathrm{c}}=P_{\mathrm{s}}+\alpha R_{\mathrm{s}}$, where $P_{\mathrm{s}}$ is the static circuit power, $\alpha$ is the dynamic circuit power per unit transmission rate, and $R_{\mathrm{S}}=R_{s_{1}}+R_{s_{2}}$ is the sum transmission rate. In order to simplify the calculation and analysis, $\alpha=0$ and the constant circuit power consumption model $P_{\mathrm{c}}=P_{\mathrm{s}}$ [19] is used in this paper.

Then, in the DT mode, the power consumption is composed of the transmit power and the circuit power as mentioned above. The duration of one time slot is denoted by $T$. Substituting $P_{\mathrm{c}}=P_{\mathrm{s}}$ into the power consumption. Consequently, the power consumption in the DT mode $P_{\mathrm{t}}$ can be calculated as

$$
\begin{aligned}
P_{\mathrm{t}} & =\frac{\left(P_{s_{1}} T+P_{s_{2}} T\right)}{2 T}+P_{\mathrm{c}} \\
& =\frac{1}{2}\left(P_{s_{1}}+P_{s_{2}}\right)+P_{\mathrm{s}} .
\end{aligned}
$$

\subsection{Power consumption in TWRT mode}

In the TWRT mode, each of the receivers at the nodes $S_{1}$ and $S_{2}$ consumes more signal processing power than that in the DT mode owing to the SIC [21]. At the same time, the relay node needs to consume the power $P_{\mathrm{r}}$ to forward signal as it mentioned above. Let $P_{\text {sic }}$ denotes the extra signal processing power for SIC. $P_{\mathrm{c}}=P_{\mathrm{s}}$ will also be substituted into the power consumption. Consequently, the power consumption in the TWRT mode $P_{\mathrm{t}}$ can be calculated as

$$
\begin{aligned}
P_{\mathrm{t}} & =\frac{\left(P_{s_{1}} T+\left(P_{s_{2}}+P_{\mathrm{r}}+2 P_{\text {sic }}\right) T\right)}{2 T}+P_{\mathrm{c}} \\
& =\frac{1}{2}\left(P_{s_{1}}+P_{s_{2}}+P_{\mathrm{r}}\right)+P_{s}+P_{\text {sic }} .
\end{aligned}
$$

\subsection{Metric of energy efficiency}

Firstly, we use $e$ to denote EE in this paper. Then, the EEs of the DT mode and the TWRT mode are denoted by $e_{\mathrm{d}}$ and $e_{\mathrm{t}}$, respectively. In order to simplify the analytical calculation, we use the ECR to evaluate EE. The ECR is the ratio of the power consumption to the sum transmission rate for 1 TTI as follows,

$$
\eta=\frac{P_{\mathrm{t}}}{R_{\mathrm{s}}}=\frac{P_{\mathrm{t}}}{R_{s_{1}}+R_{s_{2}}} .
$$

We assume that $\eta e=1$. From the relationship of $\eta$ and $e$, we can know that the denominator of the ECR can be more simple. It also can be known that the $\mathrm{EE}$ in this paper can be determined by the power consumption per unit transmission rate. It means that the transmission mode which consumes less power with the lower ECR will have a higher EE.

In practice, the transmission rates $R_{S_{1}}$ and $R_{s_{2}}$ may differ. Moreover, for $0<R_{\mathrm{S}}<\infty$, there exist various rate pairs of $R_{s_{1}}$ and $R_{s_{2}}$ that satisfy $R_{\mathrm{S}}=R_{s_{1}}+R_{s_{2}}$, but the ECRs of the DT mode and the TWRT mode with different rate pairs are different. In order to compare the ECRs of the modes and transform them into a unified form which is represented by $R_{\mathrm{s}}$, we define $R_{s_{1}}=\beta R_{\mathrm{S}}$ and $R_{s_{2}}=(1-$ $\beta) R_{\mathrm{s}}$ to reflect such an asymmetric rate scenario, where $\beta \in(0,1)[22]$ and $\beta$ is just a ratio of $R_{s_{1}}$ in $R_{\mathrm{s}}$.

\subsubsection{ECR of DT mode}

For an arbitrary $R_{\mathrm{s}}$, by minimizing $P_{\mathrm{t}}$ to minimize the ECR of the DT mode, that is to maximize the EE of the DT mode, the optimal value can be found by solving the following problem,

$$
\begin{aligned}
& P_{\mathrm{t} \text { min }}=\underbrace{\min }_{P_{s_{1}}, P_{S_{2}}}\left\{\frac{1}{2}\left(P_{s_{1}}+P_{s_{2}}\right)+P_{\mathrm{s}}\right\} \\
& \text { s.t. } R_{s_{1}}=\beta R_{\mathrm{s}} \text { and } R_{s_{2}}=(1-\beta) R_{\mathrm{s}} .
\end{aligned}
$$

Substituting $R_{s_{1}}=\beta R_{\mathrm{S}}$ and $R_{s_{2}}=(1-\beta) R_{\mathrm{S}}$ into (5) and (6), $P_{s_{1}}$ and $P_{s_{2}}$ in the DT mode can be calculated as

$$
\left\{\begin{array}{l}
P_{s_{1}}=\frac{2^{2(1-\beta) R_{\mathrm{s}}}-1}{g_{s_{1} s_{2}}}, \\
P_{s_{2}}=\frac{2^{2 \beta R_{\mathrm{s}}}-1}{g_{s_{1} s_{2}}} .
\end{array}\right.
$$

From (17), (19), and (21), the ECR of the DT mode $\eta_{\mathrm{d}}$ can be expressed as

$$
\begin{aligned}
\eta_{\mathrm{d}} & =\frac{P_{\mathrm{t}}}{R_{\mathrm{s}}}=\frac{\frac{1}{2}\left(P_{s_{1}}+P_{s_{2}}\right)+P_{\mathrm{s}}}{R_{\mathrm{s}}} \\
& =\frac{2^{2 \beta R_{\mathrm{s}}}+2^{2(1-\beta) R_{\mathrm{s}}}-2}{2 g_{s_{1} s_{2}} R_{\mathrm{s}}}+\frac{P_{\mathrm{s}}}{R_{\mathrm{s}}} .
\end{aligned}
$$

\subsubsection{ECR of TWRT mode}

To achieve a rate pair of $R_{s_{1}}$ and $R_{s_{2}}$, it is clear that we need to find $P_{s_{1}}, P_{s_{2}}$, and $P_{\mathrm{r}}$ from (15) and (16). Obviously, the 
solution is not unique, which leads to multiple choices of $P_{\mathrm{t}}$. We need to find the minimum $P_{\mathrm{t}}$ to minimize the ECR, namely to maximize the EE as mentioned above. The minimum $P_{\mathrm{t}}$ can be found by solving the following problem,

$$
\begin{aligned}
& P_{\mathrm{t} \text { min }}=\underbrace{\min }_{P_{s_{1}}, P_{s_{2}}, P_{\mathrm{r}}}\left\{\frac{1}{2}\left(P_{s_{1}}+P_{s_{2}}+P_{\mathrm{r}}\right)+P_{\mathrm{s}}+P_{\mathrm{sic}}\right\} \\
& \text { s.t. } R_{s_{1}}=\beta R_{\mathrm{s}} \text { and } R_{s_{2}}=(1-\beta) R_{\mathrm{s}} .
\end{aligned}
$$

Let us denote $c_{1}$ and $c_{2}$ as follows,

$$
c_{1}=2^{2 \beta R_{\mathrm{s}}}-1, c_{2}=2^{2(1-\beta) R_{\mathrm{s}}}-1 .
$$

Substituting $R_{s_{1}}=\beta R_{\mathrm{s}}$ and $R_{s_{2}}=(1-\beta) R_{\mathrm{s}}$ into (15) and (16), then $c_{1}$ and $c_{2}$ can be expressed as

$$
\begin{aligned}
& c_{1}=\frac{P_{\mathrm{r}} P_{s_{2}} g_{s_{1} r} g_{s_{2} r}}{P_{s_{1}} g_{s_{1} r}+\left(P_{s_{2}}+P_{\mathrm{r}}\right) g_{s_{2} r}+1}, \\
& c_{2}=\frac{P_{\mathrm{r}} P_{s_{1}} g_{s_{1} r} g_{s_{2} r}}{\left(P_{s_{1}}+P_{\mathrm{r}}\right) g_{s_{1} r}+P_{s_{2}} g_{s_{2} r}+1} .
\end{aligned}
$$

Let (25) be divided by (26), and we can get an equation of $P_{s_{1}}, P_{s_{2}}$, and $P_{\mathrm{r}}$,

$$
\begin{aligned}
& c_{2} P_{s_{2}}^{2} g_{s_{2} r}-c_{1} P_{s_{1}}^{2} g_{s_{1} r}+c_{2} P_{s_{2}} P_{s_{1}} g_{s_{1} r}-c_{1} P_{s_{1}} P_{s_{2}} g_{s_{2} r} \\
& +c_{2} P_{s_{2}}-c_{1} P_{s_{1}}=c_{1} P_{s_{1}} P_{\mathrm{r}} g_{s_{2} r}-c_{2} P_{s_{2}} P_{\mathrm{r}} g_{s_{1} r} .
\end{aligned}
$$

Then, $P_{\mathrm{r}}$ can be calculated as

$$
P_{\mathrm{r}}=\frac{\left(c_{2} P_{s_{2}}-c_{1} P_{s_{1}}\right)\left(P_{s_{1}} g_{s_{1} r}+P_{s_{2}} g_{s_{2} r}+1\right)}{\left(c_{1} P_{s_{1}} g_{s_{2} r}-c_{2} P_{s_{2}} g_{s_{1} r}\right)} .
$$

Substituting (28) into (25), we will get a equation of $P_{S_{1}}$ and $P_{s_{2}}$ as

$c_{2} P_{s_{2}}{ }^{2} g_{s_{1} r} g_{s_{2} r}+P_{s_{2}}\left(c_{1} c_{2}\left(g_{s_{1} r}-g_{s_{2} r}\right)-c_{1} P_{s_{1}} g_{s_{1} r} g_{s_{2} r}\right)=0$.

As $P_{s_{2}}>0$, from (29) we have the function

$$
P_{s_{1}}=f_{s_{1}}\left(P_{s_{2}}\right)=\frac{c_{2} P_{s_{2}} g_{s_{1} r} g_{s_{2} r}+c_{1} c_{2}\left(g_{s_{1} r}-g_{s_{2} r}\right)}{c_{1} g_{s_{1} r} g_{s_{2} r}} \text {. }
$$

Substituting (30) into (28), we have the function

$P_{\mathrm{r}}=f_{r}\left(P_{s_{2}}\right)=\frac{P_{s_{2}} g_{s_{2} r}\left(c_{1} g_{s_{2} r}+c_{2} g_{s_{1} r}\right)+c_{1} c_{2}\left(g_{s_{1} r}-g_{s_{2} r}\right)+c_{1} g_{s_{2} r}}{\left(c_{1}+P_{s_{2}}\right) g_{s_{1} r} g_{s_{2}} r^{2}}$

Then, the problem in (23) can be achieved by solving the following sub-problem

$$
\min \left(P_{s_{1}}+P_{s_{2}}+P_{\mathrm{r}}\right)=\min \left\{f_{s_{1}}\left(P_{s_{2}}\right)+P_{s_{2}}+f_{r}\left(P_{s_{2}}\right)\right\} .
$$

It is easy to see that (32) includes only one variable $P_{S_{2}}$. The optimal $P_{s_{2}}$ can be found by setting the derivative of $f\left(P_{s_{2}}\right)$ to zero. Substituting the optimal $P_{s_{2}}$ into $f_{s_{1}}\left(P_{s_{2}}\right)$ and $f_{r}\left(P_{s_{2}}\right)$, then, the transmit power $P_{s_{1}}, P_{s_{2}}$, and $P_{\mathrm{r}}$ in the TWRT mode can be calculated as

$$
\left\{\begin{array}{l}
P_{s_{1}}=\frac{c_{1}+c_{2}}{g_{s_{1} r}} \\
P_{s_{2}}=\frac{c_{1}+c_{2}}{g_{s_{2} r}} \\
P_{\mathrm{r}}=\frac{\sqrt{\left(c_{1}+c_{2}\right)\left(c_{1}+c_{2}+1\right)}}{\sqrt{g_{s_{1} r} g_{s_{2} r}}}
\end{array}\right.
$$

By substituting (24) into (33), the minimum $P_{\mathrm{t}}$ in the TWRT mode can be expressed as

$$
\begin{aligned}
P_{\mathrm{tmin}}= & \underbrace{\min }_{P_{s_{1}}, P_{s_{2}}, P_{\mathrm{r}}}\left\{\frac{1}{2}\left(P_{s_{1}}+P_{s_{2}}+P_{\mathrm{r}}\right)+P_{\mathrm{s}}+P_{\mathrm{sic}}\right\} \\
= & \frac{2^{2 \beta R_{\mathrm{s}}}+2^{2(1-\beta) R_{\mathrm{s}}}-2}{2}\left(\frac{1}{g_{s_{1} r}}+\frac{1}{g_{s_{2} r}}\right) \\
& +\frac{\sqrt{\left(2^{2 \beta R_{\mathrm{s}}}+2^{2(1-\beta) R_{\mathrm{s}}}-2\right)\left(2^{2 \beta R_{\mathrm{s}}}+2^{2(1-\beta) R_{\mathrm{s}}}-1\right)}}{\sqrt{g_{s_{1} r} g_{s_{2} r}}} \\
& +P_{\mathrm{s}}+P_{\text {sic }} .
\end{aligned}
$$

From (19) and (34), the ECR of the TWRT mode $\eta_{\mathrm{t}}$ can be expressed as

$$
\begin{aligned}
\eta_{\mathrm{t}} & =\frac{P_{\mathrm{t}}}{R_{\mathrm{s}}}=\frac{2^{2 \beta R_{\mathrm{s}}}+2^{2(1-\beta) R_{\mathrm{s}}}-2}{2 R_{\mathrm{s}}}\left(\frac{1}{g_{s_{1} r}}+\frac{1}{g_{s_{2} r}}\right) \\
+ & \frac{\sqrt{\left(2^{2 \beta R_{\mathrm{s}}}+2^{2(1-\beta) R_{\mathrm{s}}}-2\right)\left(2^{2 \beta R_{\mathrm{s}}}+2^{2(1-\beta) R_{\mathrm{s}}}-1\right)}}{\sqrt{g_{s_{1} r} g_{s_{2} r}} R_{\mathrm{s}}} \\
+ & \frac{P_{\mathrm{s}}+P_{\mathrm{sic}}}{R_{\mathrm{S}}} .
\end{aligned}
$$

\section{Comparison and analysis of energy efficiency 4.1 Comparison of energy efficiency} In this section, the ECRs of the DT mode and the TWRT mode will be compared to get a comparison between $e_{\mathrm{d}}$ and $e_{t}$. An AWT strategy will also be designed to achieve the minimize ECR, that is to achieve the maximum EE.

The difference of ECRs between the DT mode and the TWRT mode $f\left(R_{\mathrm{s}}\right)$ can be expressed as follows, i.e.,

$$
f\left(R_{\mathrm{s}}\right)=\eta_{\mathrm{d}}-\eta_{\mathrm{t}}
$$

From (34), it is easy to see that $P_{\mathrm{t}}$ in the TWRT mode is very complicated. However, when $R_{\mathrm{s}}$ is large enough such that the following approximation can be used

$$
2^{2 \beta R_{\mathrm{s}}}+2^{2(1-\beta) R_{\mathrm{s}}}-2 \approx 2^{2 \beta R_{\mathrm{s}}}+2^{2(1-\beta) R_{\mathrm{s}}}-1 .
$$


Substituting (37) into (36), then $f\left(R_{\mathrm{s}}\right)$ can be expressed as

$$
\begin{aligned}
f\left(R_{\mathrm{s}}\right)= & \frac{2^{2 \beta R_{\mathrm{s}}}+2^{2(1-\beta) R_{\mathrm{s}}}-2}{2 R_{\mathrm{s}}}\left[\frac{1}{g_{s_{1} s_{2}}}-\left(\frac{1}{\sqrt{g_{s_{1} r}}}+\frac{1}{\sqrt{g_{s_{2} r}}}\right)^{2}\right] \\
& -\frac{P_{\text {sic }}}{R_{\mathrm{s}}} .
\end{aligned}
$$

We assume that $\theta=\frac{1}{g_{s_{1} s_{2}}}-\left(\frac{1}{\sqrt{g_{s_{1} r}}}+\frac{1}{\sqrt{g_{s_{2} r}}}\right)^{2}$, then, when $\theta \leq 0, f\left(R_{\mathrm{s}}\right)<0$ as $\frac{P_{\text {sic }}}{R_{\mathrm{s}}}>0$. Otherwise, $\theta>0$, then, there is a uncertainty about which one of $\eta_{\mathrm{d}}$ and $\eta_{\mathrm{t}}$ is the larger. However, it can be seen that when $f\left(R_{\mathrm{s}}\right)=0$, there is a threshold $R_{\text {th }}$ which makes the ECRs of the two kinds of transmission modes equal, that is, makes the EEs of the DT mode and the TWRT mode equal. With the threshold $R_{\text {th }}$, we can design an AWT strategy to decrease the $\mathrm{ECR}$, namely as mentioned above to improve the EE of the wireless transmission system. The threshold $R_{\text {th }}$ will be considered in the next section.

\subsection{Analysis of energy efficiency}

The threshold $R_{\text {th }}$ can be obtained when $f\left(R_{\mathrm{s}}\right)=0$. From (38) we can get the following equation

$$
2^{2 \beta R_{\mathrm{s}}}+2^{2(1-\beta) R_{\mathrm{s}}}-2=\frac{2 P_{\text {sic }}}{\theta} .
$$

We assume that $2^{2 R_{\mathrm{s}}}=x$, and $\frac{2 P_{\text {sic }}}{\theta}=Q$. From (39), we can define a function with respect to $\beta$ as follows

$$
\begin{aligned}
f(\beta) & =x^{\beta}+x^{(1-\beta)} \\
& =Q+2 .
\end{aligned}
$$

We take the derivative of $f(\beta)$, then $f^{\prime}(\beta)=\left(x^{\beta}-\right.$ $\left.x^{(1-\beta)}\right) \ln x$. Since $R_{\mathrm{S}}>0$, then $x>1$ and $\ln x>0$. We can obtain $f^{\prime}(\beta)<0$ when $\beta \in(0,0.5)$, and $f^{\prime}(\beta)>0$ when $\beta \in(0.5,1)$. The minimum value of the threshold $R_{\text {th }}$ can be found by setting the derivative of $f(\beta)$ to zero, i.e., $\beta=0.5$. After that, the range of $f(\beta)$ is

$$
2 \sqrt{x} \leq f(\beta) \leq x+1,
$$

which implies $2 \sqrt{x} \leq Q+2 \leq x+1$. Finally, the range of $R_{\text {th }}$ can be computed as

$$
\frac{1}{2} \log _{2}(Q+1) \leq R_{\text {th }} \leq \log _{2} \frac{Q+2}{2} .
$$

Next, the range of $Q$ will also be considered to investigate the effect with the location of the relay node $R$. According to [23], the channel transmission model can be expressed as

$$
\bar{H}_{a b}=k\left(d_{a b}\right)^{-c},
$$

where $\bar{H}_{a b}$ is the mean of channel gain $H_{a b}, k$ is a constant determined by the communication environment, $d_{a b}$ is the distance between the node $a$ and $b$, and $c$ is the path loss exponent and usually $c>2$ [24]. Compared with the large-scale fading of the relay location, we are going to ignore the Rayleigh fading in the analysis which simplifies the mathematical analysis [23], thus $Q$ can be expressed in terms of the inter-node distance and the channel gain is proportional to $\left(d_{a b}\right)^{-c}$. In order to simplify the calculation, we assume that $k=1$. As it is shown in Fig. 1b, $d_{s_{1} s_{2}}=d_{s_{1} r}+d_{s_{2} r}$. We also assume that $d_{s_{1} s_{2}}=1$ and $d_{s_{1} r}=d$, then $d_{s_{2} r}=1-d$. The denominator of $Q$ is $\theta$, from the assumptions of $g_{s_{1} s_{2}}, g_{s_{1} r}$, and $g_{s_{2} r}$, then $\theta$ can be expressed as

$$
\theta=\sigma^{2}\left(1-\left((1-d)^{\frac{c}{2}}+d^{\frac{c}{2}}\right)^{2}\right) .
$$

From (42), we can know that the threshold $R_{\text {th }}$ is a monotone increasing function of $Q$ and $P_{\text {sic }}$ is a constant. Then, the range of $Q$ mainly depends on the value of $\theta$. Since $N=(1-d)^{\frac{c}{2}}+d^{\frac{c}{2}} \geq 2 \sqrt{(1-d)^{\frac{c}{2}} d^{\frac{c}{2}}}$, the lower bound can be obtained as $N \geq 2^{1-\frac{c}{2}}$ when $d=0.5$. The upper bound can be obtained as $N<1$ when $d$ is infinitely approximate to 0 or 1 . The range of $\theta$ can be found as follows

$$
0<\theta \leq \sigma^{2}\left(1-2^{2-c}\right) .
$$

Consequently, the range of $Q$ can be calculated as

$$
\frac{2 P_{\text {sic }}}{\sigma^{2}\left(1-2^{2-c}\right)} \leq Q<\infty .
$$

Substituting (46) into (42), the range of the threshold $R_{\text {th }}$ which makes the ECRs of the DT mode and the TWRT mode equal can be determined.

Based on the analysis above, it is clear that the threshold $R_{\mathrm{th}}$ is just a range of transmission rate. The threshold $R_{\text {th }}$ varies with $\beta$ which is also a variable rather than a certain value. Therefore, the certain threshold $R_{\text {th }}$ cannot be found. From the perspective of a certain threshold $R_{\mathrm{th}}$, the TRTD algorithm with the bisection method which is offline will be used to find the threshold $R_{\mathrm{th}}$. This TRTD algorithm is presented in Algorithm 1.

With the TRTD algorithm, the threshold $R_{\text {th }}$ will be found. Then, we can design the AWT strategy to achieve an energy-efficient adaptive transmission. The ECR of the AWT strategy is defined by $\eta_{\mathrm{a}}$ and $\eta_{\mathrm{a}}=\min \left\{\eta_{\mathrm{d}}, \eta_{\mathrm{t}}\right\}$. In the AWT strategy, at first the wireless transmission system is working in the DT mode. When $\theta \leq 0$, then $\eta_{\mathrm{a}}=\eta_{\mathrm{d}}$, and the system keeps working in the DT mode. Otherwise, when $\theta>0$, then, we compare $R_{\mathrm{S}}$ and $R_{\text {th }}$ according to Algorithm 1. If $R_{\mathrm{s}} \leq R_{\mathrm{th}}$, and $\eta_{\mathrm{a}}=\eta_{\mathrm{d}}$, the system still keeps working in the DT mode. The system will change its working mode to the TWRT mode unless $\theta>0, R_{\mathrm{s}}>R_{\mathrm{th}}$, and $\eta_{\mathrm{a}}=\eta_{\mathrm{t}}$. 


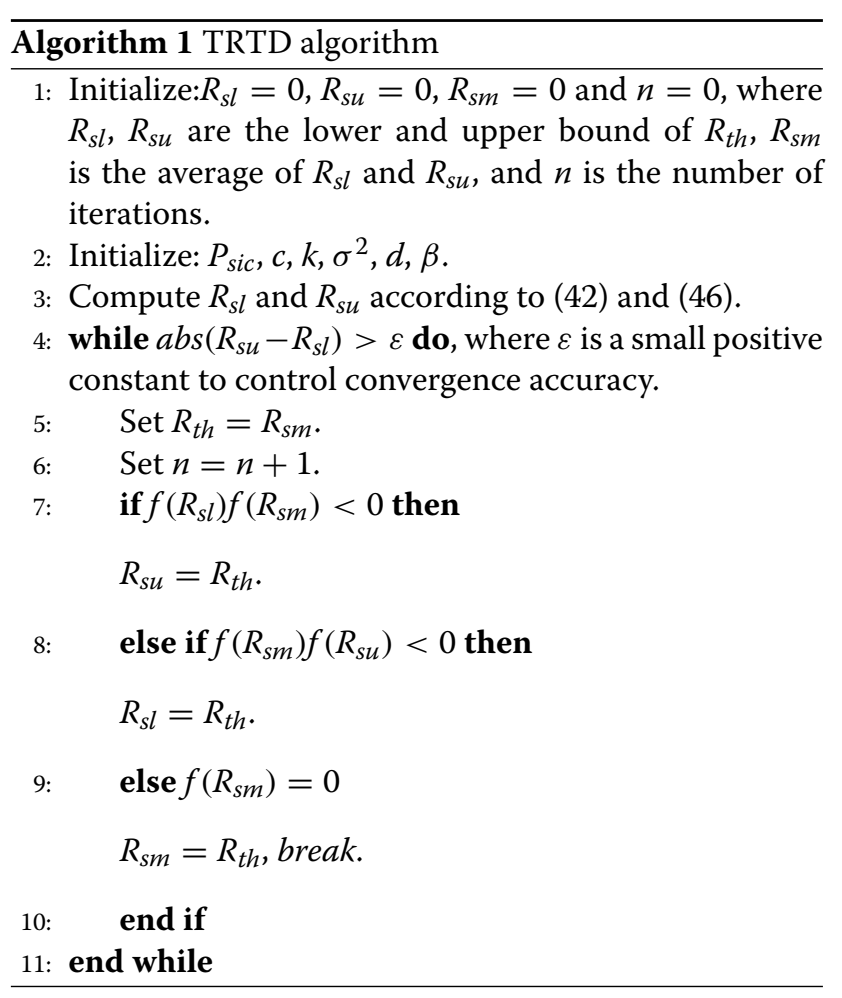

From the AWT strategy, we can know that when $f\left(R_{\mathrm{s}}\right) \leq$ 0 , the transmission mode of the wireless transmission system will be the DT mode and the relay node is in a state of sleep. When $f\left(R_{\mathrm{s}}\right)>0$, the transmission mode of the wireless transmission system will be the TWRT mode and the source node sends a request (REQ) to the relay node. The relay node changes it state into active and meanwhile the relay node sends an acknowledgement (ACK) to the source node. Then, the TWRT mode will be set up. With the AWT strategy, the ECR is the minimum, then the EE of the wireless transmission system is always the maximum at a range of transmission rate.

\section{Simulation results}

In this section, in order to confirm the validity of the analytical expressions, the simulation results are conducted. The EE of the AWT strategy is denoted by $e_{\mathrm{a}}$, where $e_{\mathrm{a}}=$ $\max \left\{e_{\mathrm{d}}, e_{\mathrm{t}}\right\}$. To evaluate and compare the EEs with various transmission modes, without loss of generality, the Rayleigh fading channel and the AWGN model is considered in the Monte Carlo simulation. It is assumed that $c=4$, and the noise variance is 1 .

\subsection{EE comparison with ideal and practical power systems}

As a baseline for comparison, we first compare the EEs of the DT mode and the TWRT mode with consideration of the ideal and the practical power system. In the ideal power system, $P_{\mathrm{s}}=0$. In the practical power system, $P_{\mathrm{s}}=$

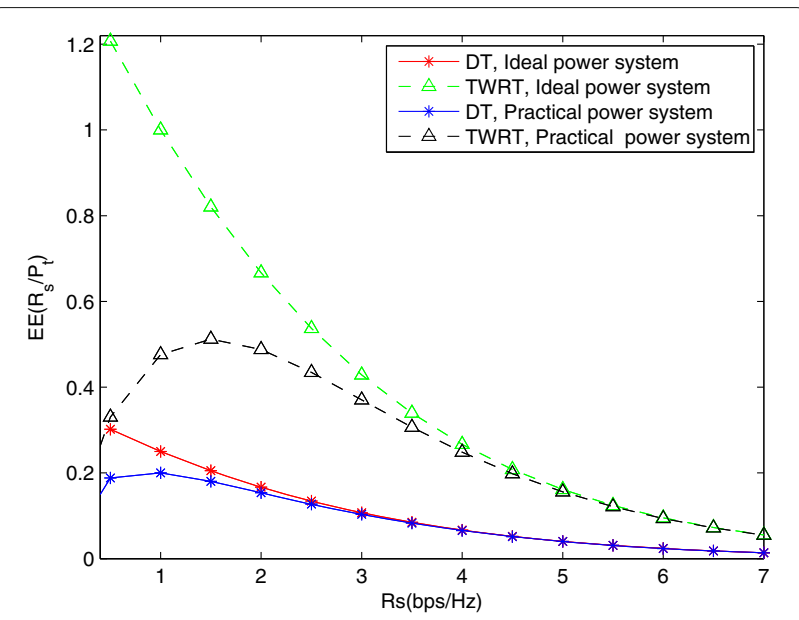

Fig. 2 EE comparison between ideal and practical power system

$1 \mathrm{~W}$ and $P_{\text {sic }}=0.1 \mathrm{~W}$. The other simulation parameters are given as $\beta=0.5$ and $d=0.5$.

In Fig. 2, the following observations are obtained. (i) The EEs of the TWRT mode are always better than that of the DT mode. This shows the effectiveness of the relay technique in increasing the capacity and reducing the power consumption. (ii) When $R_{\mathrm{s}}$ is low, the EEs of the ideal power system are always better than that of the practical power system, both in the DT mode and the TWRT mode. This implies the importance to consider the practical power consumption since it influences EE greatly and cannot be ignored.

\subsection{EE comparison with effects of different parameters}

In this subsection, the EE comparison with the effects of different parameters will be presented based on the theories mentioned above.

In Fig. 3, the EE comparison with the effect of $P_{\mathrm{s}}$ are depicted. The simulation parameters are $\beta=0.5, d=0.5$, and $P_{\text {sic }}=0.1 \mathrm{~W}$. The $P_{\mathrm{s}}$ are $0.1,1$, and $10 \mathrm{~W}$, respectively. It can be seen from Fig. 3 that the EEs firstly increase with the increasing of $R_{\mathrm{s}}$ and then decrease. This is because for $R_{\mathrm{S}} \in(0,7)$, there is a point which makes $\frac{R_{\mathrm{s}}}{P_{\mathrm{t}}}$ the maximum and this is determined by the ECR. The EEs also gradually become worse with the increasing of $P_{\mathrm{s}}$ both in the DT mode and the TWRT mode. It demonstrates the necessity to consider $P_{\mathrm{s}}$ in the wireless transmission system.

In Fig. 4, the EE comparison with the effect of $P_{\text {sic }}$ are depicted. The simulation parameters are $\beta=0.5, d=0.5$, and $P_{\mathrm{s}}=0.1 \mathrm{~W}$. The $P_{\text {sic }}$ are $0.1,1$, and $10 \mathrm{~W}$, respectively. It can be seen from Fig. 4 that the EEs of the TWRT mode gradually become worse with the increasing of $P_{\text {sic }}$ and the EEs of the TWRT mode are not always better than that of the DT mode. We can know that when $P_{\text {sic }}$ is big enough, the effectiveness of the relay technique will also be offset, and it demonstrates the significance of $P_{\text {sic }}$. 


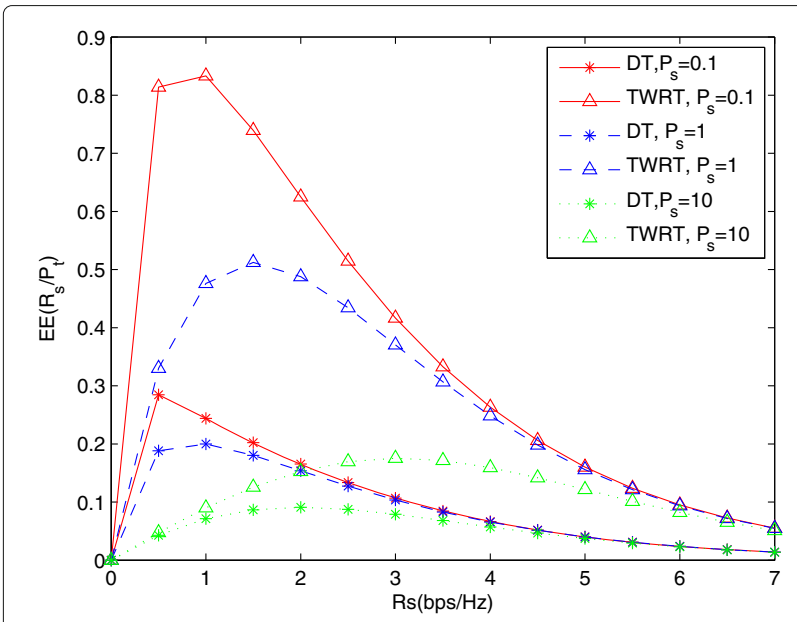

Fig. 3 EE comparison with the effect of $P_{\mathrm{s}}$

In Fig. 5, the EE comparison with the effect of $\beta$ are depicted. The simulation parameters are given as $d=0.5$, $P_{\mathrm{s}}=0.1 \mathrm{~W}$, and $P_{\text {sic }}=0.1 \mathrm{~W}$. In the DT mode, $\beta$ is 0.5 , and in the TWRT mode, $\beta$ are 0.5 and 0.9 , respectively. It is shown in Fig. 5 that the EE of the DT mode is even better than that of the TWRT mode when $R_{\mathrm{S}}$ is high and $\beta=0.9$. The EE of the TWRT mode when $\beta=0.5$ reaches the best status. It shows the significance of the value with $\beta$. It means that when the rate pair of $R_{s_{1}}$ and $R_{S_{2}}$ are equal, namely the rate task of the source nodes $S_{1}$ and $S_{2}$ are equal, we will get the maximum EE of the system. This is because in such situation, the channel conditions between any two nodes will not be bad and the relay can forward the signal effectively.

In Fig. 6, the EE comparison with the effect of $d$ are depicted. The simulation parameters are given as $\beta=0.5$, $P_{\mathrm{s}}=1 \mathrm{~W}$, and $P_{\text {sic }}=0.1 \mathrm{~W} . d$ are 0.5 and 0.8 , respectively.

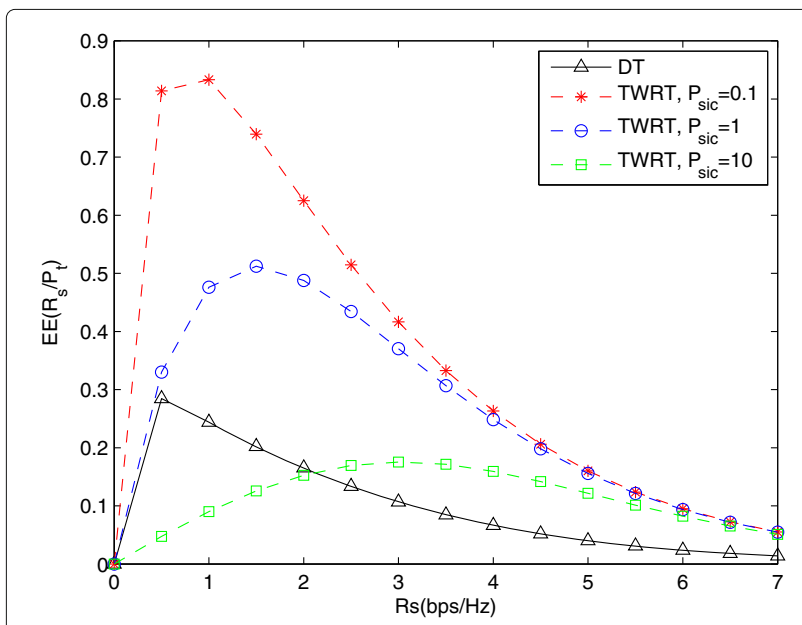

Fig. 4 EE comparison with the effect of $P_{\text {sic }}$

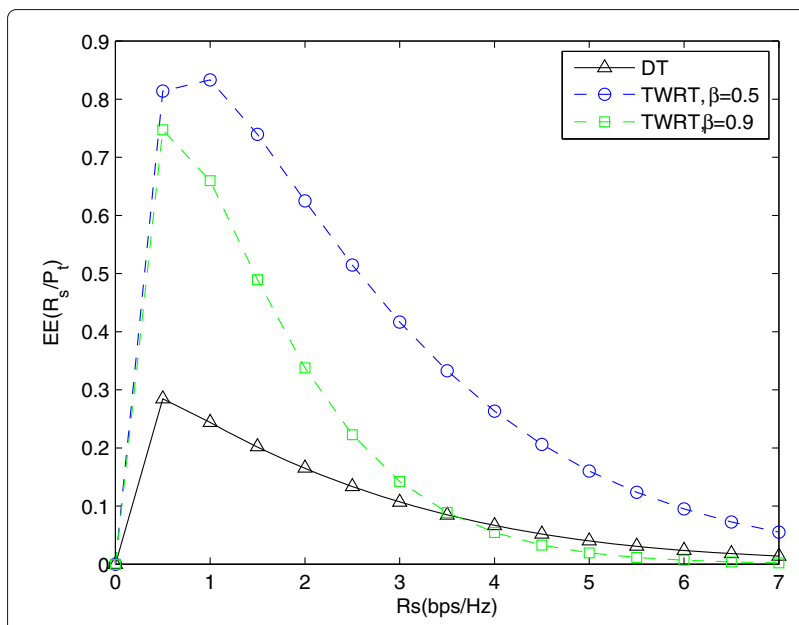

Fig. 5 EE comparison with the effect of $\beta$

It is shown in Fig. 6 that the EE of the TWRT mode when $d=0.8$ (the relay node is closer to the node $S_{2}$ ) is better than that of the DT mode. The effectiveness of the relay technique can still be shown. The EE of the TWRT mode when $d=0.5$ (the relay node is just in the middle of the node $S_{1}$ and the node $S_{2}$ ) is the best. This is because when the relay node is in the middle of the two nodes, the relay node can maximize its advantages of increasing the capacity and reducing the power consumption. It confirms the significance of optimizing the location of the relay node.

\subsection{EEs comparison with various transmission schemes}

In this subsection, the EEs comparison with various transmission schemes are provided. The simulation parameters are $\beta=0.9, d=0.5, P_{\mathrm{s}}=1 \mathrm{~W}$, and $P_{\text {sic }}=1 \mathrm{~W}$.

It is shown in Fig. 7 that the EE of the TWRT mode is not always better than that of the DT mode when $R_{\mathrm{s}}$

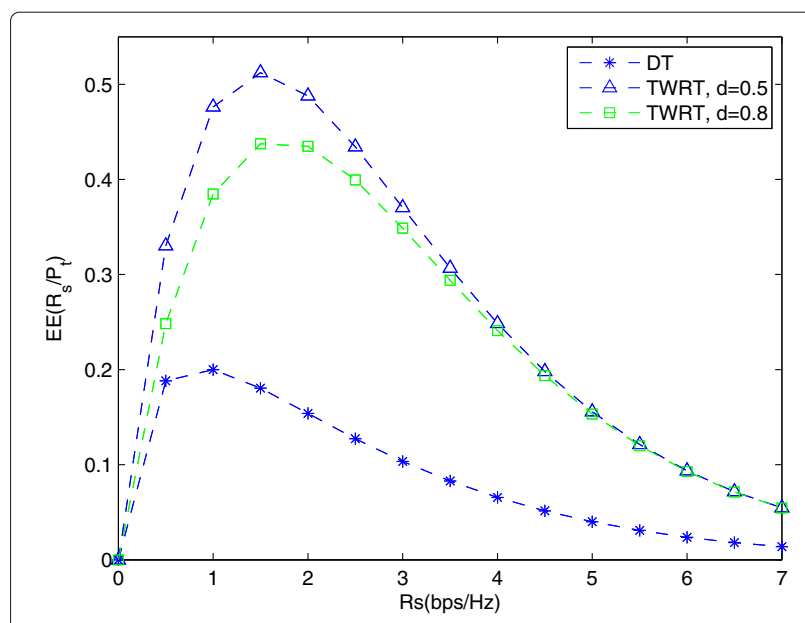

Fig. 6 EE comparison with the location of relay 
is low. This is because the relay node $R$ consumes the power to forward the noise for the unreasonable rate pairs with $\beta=0.9$. It also can be seen that when $R_{\mathrm{s}}$ is about $3.5(\mathrm{bps} / \mathrm{Hz})$, the EEs of the DT mode and the TWRT mode are equal. Obviously, the threshold $R_{\mathrm{th}}=$ $3.5(\mathrm{bps} / \mathrm{Hz})$. With the AWT strategy when $R_{\mathrm{s}}$ is smaller than or is equal to $3.5(\mathrm{bps} / \mathrm{Hz})$, the $\mathrm{EE}$ is equal to that of the DT mode. But when $R_{\mathrm{s}}$ is higher than $3.5(\mathrm{bps} / \mathrm{Hz})$, the EE is equal to the one of the TWRT mode. This is because the EE of our AWT strategy is the maximum of $e_{\mathrm{d}}$ and $e_{\mathrm{t}}$ and all these results are in accordance with our analysis.

It can be seen from the simulation results that the theoretical analysis is correct. The EE is closely related with the relevant parameters, such as $P_{\mathrm{c}}, P_{\mathrm{sic}}, \beta$, and $d$. The EE in our AWT strategy is always the maximum at a range of transmission rate. Evidently, it demonstrates the effectiveness of our proposed AWT strategy.

\section{Conclusions}

In this paper, to improve EE, we have proposed an AWT strategy with consideration of the circuit power in which the transmission mode was switched between the DT mode and the TWRT mode. The switch strategy was based on a transmission rate threshold, which made the EEs of the DT mode and the TWRT mode equal. The TRTD algorithm with a bisection method has been used to find the threshold. The analytical and simulation results have also shown that our AWT strategy was more efficient at a reasonable range of transmission rate. Future work can consider the position of the relay node more specifically and practically. The two-way full-duplex model to improve EE and SE at the same time can also be considered.

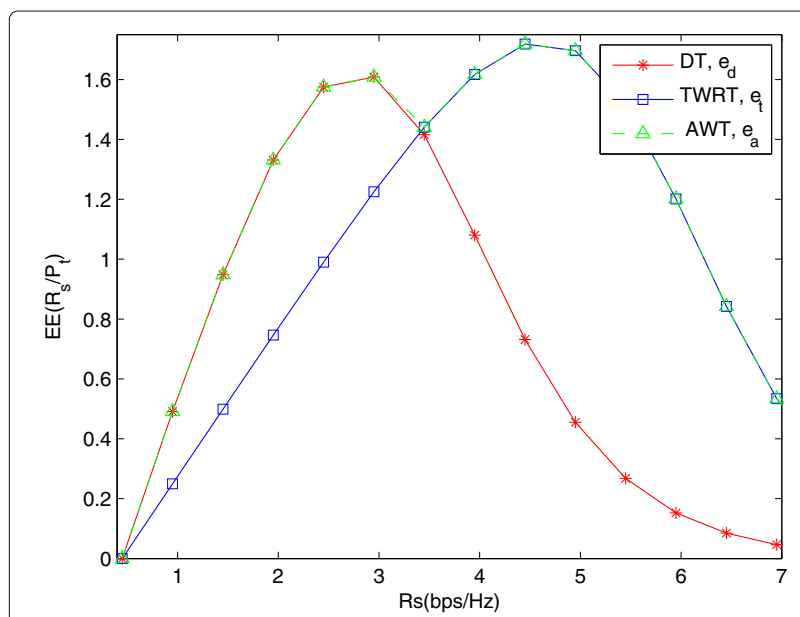

Fig. 7 EE comparison with various transmission modes

\section{Acknowledgements}

This work was sponsored by the Shanghai Rising-Star Program (15QA1400100) Innovation Program of Shanghai Municipal Education Commission (15ZZ03), and DHU Distinguished Young Professor Program (16D210402).

\section{Competing interests}

The authors declare that they have no competing interests.

Received: 4 July 2016 Accepted: 2 January 2017

Published online: 21 January 2017

\section{References}

1. S Bayhan, F Alagoz, Scheduling in centralized cognitive radio networks for energy efficiency. IEEE Trans. Veh. Technol. 62, 582-595 (2013)

2. M Mhiri, VS Varma, K Cheikhrouhou, L Lasaulce, A Samet, Cross-layer distributed power control: a repeated game formulation to improve the sum energy efficiency. EURASIP. Wirel. Commun. Net. 257, 1-6 (2015)

3. GY Li, Z Xu, C Xiong, C Yang, S Zhang, Y Chen, S Xu, Energy-efficient wireless communications: tutorial, survey, and open issues. IEEE Trans. Wirel. Commun. 18, 28-35 (2011)

4. M Zhou, Q Cui, M Valkama, X Tao, Energy-efficient resource allocation for OFDMA based two-way relay channel with physical-layer network coding. EURASIP. Wirel. Commun. Net. 66, 1-11 (2012)

5. N Abuzainab, A Ephremides, Energy efficiency of cooperative relaying over a wireless link. IEEE Trans. Wirel. Commun. 11, 2076-2083 (2012)

6. CH Liu, LC Wang, Optimal cell load and throughput in green small cell networks with generalized cell association. IEEE J. Sel. Areas Commun. 34 1058-1072 (2016)

7. K Wang, Y Chen, MS Alouini, F Xu, BER and optimal power allocation for amplify-and-forward relaying using pilot-aided maximum likelihood estimation. IEEE Trans. Wirel. Commun. 62, 3462-3475 (2014)

8. Y Wang, P Ren, Q Du, L Sun, Optimal power allocation for underlay-based cognitive radio networks with primary user's statistical delay QoS provisioning. IEEE Trans. Wirel. Commun. 14, 6896-6910 (2015)

9. Y Rong, Joint source and relay optimization for two-way linear non-regenerative MIMO relay communications. IEEE Trans. Signal Process 60, 6533-6546 (2012)

10. $\mathrm{X} \mathrm{Ji}, \mathrm{B}$ Zheng, Y Cai, L Zou, On the study of half-duplex asymmetric two-way relay transmission using an amplify-and-forward relay. IEEE Trans. Veh. Technol. 61, 1649-1664 (2012)

11. B Rankov, A Wittneben, Spectral efficient protocols for half duplex fading relay channels. IEEE J. Sel. Areas Commun. 25, 379-389 (2007)

12. AH El-Malke Abd, SA Zummo, in Proc. 2015 IEEE 82nd Vehicular Technology Conference (VTC2015-Fall). A cooperative model for enhancing spectral efficiency in two-way amplify-and-forward relaying networks, (Boston, MA, 2015), pp. 1-5

13. CXiong, L Lu, GY Li, Energy-efficient OFDMA-based two-way relay. IEEE Trans. Commun. 63, 3157-3169 (2015)

14. K Singh, ML Ku, JC Lin, in Proc. 2015 IEEE 26th Annual International Symposium on Personal, Indoor, and Mobile Radio Communication (PIMRC). Joint QOS-promising and EE-balancing power allocation for two-way relay networks, (Hongkong, China, 2015), pp. 1781-1785

15. Z Chang, Q Zhang, X Guo, Z Zhou, T Ristantiemi, in Proc. 2015 IEEE Military Communication Conference( MILCOM 2015). Energy efficient resource allocation for OFDMA two-way relay networks with channel estimation error, (Tampa, FL, 2015), pp. 559-564

16. Q Cui, T Yuan, X Tao, AA Dowhuszko, R Jantti, Energy efficiency analysis of two-way DF relay system with non-ideal power amplifiers. IEEE Commun. Lett. 18, 1254-1257 (2014)

17. TE D Persson, EG Larsson, Amplifier-aware,multiple-input,multiple-output power allocation. IEEE Commun. Lett. 17, 1112-1115 (2013)

18. Y Li, Z Zheng, M Zhao, Y Chen, C Liu, Energy efficient design for two-way AF relay networks. Inter. J. Anten. Propa. 292087, 1-6 (2014)

19. G Miao, N Himayat, GY Li, Energy-efficient hybrid one- and two-way relay transmission. IEEE Trans. Commun. 58, 545-554 (2010)

20. C Xiong, GY Li, S Zhang, Y Chen, S Xu, Energy-and spectral-efficiency tradeoff in down link OFDMA networks. IEEE Trans. Wirel. Commun. 10 3874-3886 (2011)

21. C Sun, C Yang, Energy-efficient hybrid one- and two-way relay transmission. IEEE Trans. Veh. Technol. 62, 3737-3751 (2013) 
22. C Sun, C Yang, in Proc. GLOBECOM'11. Is two-way relay more energy efficient?, (Houston, TX, USA, 2011), pp. 1-6

23. Q Sun, L Li, M Juntti, in Proc. WCNC'13. Energy Efficient Transmission and Optimal Relay Location for Two-Way Relay Systems, (Shanghai, China, 2013), pp. 2828-2832

24. C Liu, R Rong, S Cui, Optimal discrete power control in poisson-clustered ad hoc networks. IEEE Trans. Wirel. Commun. 14, 138-151 (2015)

\section{Submit your manuscript to a SpringerOpen ${ }^{\circ}$} journal and benefit from:

- Convenient online submission

- Rigorous peer review

- Immediate publication on acceptance

- Open access: articles freely available online

- High visibility within the field

- Retaining the copyright to your article

Submit your next manuscript at springeropen.com 